\title{
Comparison of long-term decentration and tilt in two types of multifocal intraocular lenses with OPD-Scan III aberrometer
}

\author{
Wenwen $\mathrm{He}^{1,2,3} \cdot$ Xiaodi Qiu ${ }^{1,2,3} \cdot$ Shaohua Zhang ${ }^{1,2,3} \cdot \mathrm{Yu} \mathrm{Du}^{1,2,3} \cdot$ Yinglei Zhang ${ }^{1,2,3} \cdot \mathrm{Yi} \mathrm{Lu}^{1,2,3} \cdot$ Xiangjia Zhu $^{1,2,3}$
}

Received: 31 August 2017 / Revised: 8 January 2018 / Accepted: 8 February 2018 / Published online: 9 March 2018

(C) The Royal College of Ophthalmologists 2018

\begin{abstract}
Purpose To compare the long-term decentration and tilt in two multifocal intraocular lenses (MfIOLs) with an OPD-Scan III aberrometer.

Methods Eighty cataract patients who underwent uneventful MfIOL implantation (42 with AcrySof ${ }^{\circledR}$ IQ ReSTOR ${ }^{\circledR}$ SN6AD1 and 38 with AMO Tecnis ${ }^{\circledR}$ ZMB00) were enrolled. At 1 year after surgery, a postoperative visual acuity evaluation included the measurement of uncorrected distance visual acuity, corrected distance visual acuity, distance-corrected near visual acuity, and distance-corrected intermediate visual acuity. OPD-Scan III aberrometer was used to collect the decentration, tilt, and high-order aberration (HOA) data. Significance was tested with Student's $t$ test, Mann-Whitney $U$ test, paired $t$ test, and $\chi^{2}$ test

Results The average intraocular tilt was lower in the SN6AD1 group than in the ZMB00 group, whereas the average decentration of the two groups did not differ significantly. The mean total ocular HOAs, ocular trefoil, total internal HOAs, and spherical aberrations were significantly lower in the SN6AD1 group than in the ZMB00 group. In both the SN6AD1 and ZMB00 groups, the intraocular tilt was directly proportional to the total ocular HOAs, coma, and spherical aberration. However, there was no significant correlation between decentration and any type of HOA in SN6AD1 group, whereas decentration correlated positively with total ocular HOAs, coma, and spherical aberration in ZMB00 group.

Conclusion Both MfIOLs significantly improve the visual acuity of the patients. However, tilt was lower in the SN6AD1 group than in the ZMB00 group 1 year after surgery, which provide fewer HOAs and better visual quality.
\end{abstract}

\section{Introduction}

With innovations in surgical techniques and concepts, cataract surgery has recently become a type of refractive

\footnotetext{
These authors contributed equally: W He, X Qiu

Yi Lu

luyieent@126.com

$\triangle$ Xiangjia Zhu

zhuxiangjia1982@126.com

1 Eye Institute,Eye and Ear, Nose, and Throat Hospital Fudan University, 83 Fenyang Road, Shanghai 200031, People's Republic of China

2 Key Laboratory of Myopia, Ministry of Health, Shanghai 200031, People's Republic of China

3 Shanghai Key Laboratory of Visual Impairment and Restoration, Shanghai 200031, People's Republic of China
}

surgery. Patients have better visual and refractive outcomes, exceeding mere visual restoration. Cataract surgeons aim to improve the patient's quality of life by achieving spectacle independence over all distances. Previous studies have shown that multifocal intraocular lenses (MfIOLs) can provide satisfactory near vision for these patients, at the cost of slightly reduced retinal image contrast [1-3]. However, the decentration and tilt of MfIOLs may significantly damage their optical quality, and consequently affect the patient's quality of life.

AcrySof ${ }^{\circledR}$ IQ ReSTOR ${ }^{\circledR}$ SN6AD1 (Alcon Laboratories, Inc., Irvine, CA, USA) and AMO Tecnis ${ }^{\circledR}$ ZMB00 (Abbott Medical Optics, Santa Ana, CA, USA) are two widely used MfIOLs. Although several studies have compared the visual quality and distant and near vision afforded by SN6AD1 and ZMB00 [4, 5], there has been no comparison of the long-term central stability of these two MfIOLs after implantation.

The generally accepted method for evaluating the decentration and tilt of intraocular lenses (IOLs) is the 
Purkinje imaging method [6] or Scheimpflug image processing [7], both of which require image processing software and complex calculations. The new-generation corneal topographer, OPD-Scan III (Nidek Co., Ltd., Gamagori, Japan), simplifies this problem by directly providing the tilt data based on aberration calculations in one measurement $[8,9]$, and the eccentricity of the IOLs through retroillumination. The purpose of this study was to transversally compare the long-term decentration and tilt of SN6AD1 and ZMB00 MfIOLs with an OPD-Scan III aberrometer.

\section{Subjects and methods}

\section{Patients}

This transversal study based on partial retrospective data was approved by the Ethics Committee of the Eye and ENT Hospital of Fudan University, Shanghai, China. Informed consent was obtained from all the participants. We reviewed the medical charts of cataract patients who had undergone uneventful phacoemulsification and MfIOL implantation between January 2015 and December 2015 at the Eye and ENT Hospital of Fudan University. The inclusion criteria were: (1) a diagnosis of age-related cataract, with corneal astigmatism of $\leq 1.00$ cylindrical diopter, pupil diameter of $>3.0 \mathrm{~mm}$ under mesopic conditions, and a kappa angle within the normal range; and (2) phacoemulsification combined with ZMB00 or SN6AD1 IOL implantation. The exclusion criteria were: (1) presence of any other eye pathology or neuropathy, such as corneal disease, glaucoma, trauma, or fundus pathology, that could reduce visual acuity; (2) intra- or postoperative complications, such as posterior capsule rupture, failure of continuous circular capsulorhexis (CCC), or severe posterior capsular opacification, with or without YAG laser treatment; and (3) eyes with axial lengths longer than $26 \mathrm{~mm}$ or shorter than 20 $\mathrm{mm}$. In total, 80 eyes of 80 patients were enrolled: 42 eyes of 42 patients were implanted with SN6A1 and 38 eyes of 38 patients were implanted with ZMB00.

\section{Preoperative examinations}

The ophthalmic examinations performed before surgery included an assessment of uncorrected visual acuity (logarithm of the minimal angle of resolution [logMAR]), slitlamp microscopy, fundus examination, corneal endothelium count, corneal topography (Pentacam HR; Oculus Optikgeräte, Wetzlar, Germany), B mode ultrasound scan, and measurement with IOL Master 500 (Carl Zeiss AG, Oberkochen, Germany).

\section{Surgical technique}

All surgery was performed by one experienced doctor (YL), using the same procedure. A $2.6 \mathrm{~mm}$ temporal clear corneal incision was made, followed by injection of viscoelastic material into the anterior chamber. Phacoemulsification was applied after the $5.5 \mathrm{~mm} \mathrm{CCC}$ and hydrodissection were completed. The ZMB00 or SN6AD1 MfIOL was implanted in the capsular bag. After the viscoelastics were removed, the IOL was adjusted to the center, and the incision was hydrated. No stitches were used in any eye. The postoperative medications given were levofloxacin and prednisolone acetate, four times/day for 2 weeks, and pranoprofen, four times/day for 4 weeks.

\section{1-Year postoperative examination}

One year after surgery, all patients received routine postoperative examinations. Uncorrected distance visual acuity (UDVA), best corrected distance visual acuity, uncorrected near visual acuity (UNVA), and computer and subjective refraction were recorded under photopic $\left(85 \mathrm{~cd} / \mathrm{m}^{2}\right)$ lighting. An OPD-Scan III aberrometer provided the total and intraocular high-order aberration (HOA) data with a mesopic pupil under mesopic $\left(3 \mathrm{~cd} / \mathrm{m}^{2}\right)$ lighting conditions. The tilt of the IOL was reported as an intraocular tilt datum. In the retroillumination analysis mode, the instrument identifies the center of the visual axis and the diffraction ring in multifocal IOLs (Fig. 1). The distance from the center of the diffraction ring to the center of the visual axis was then measured.

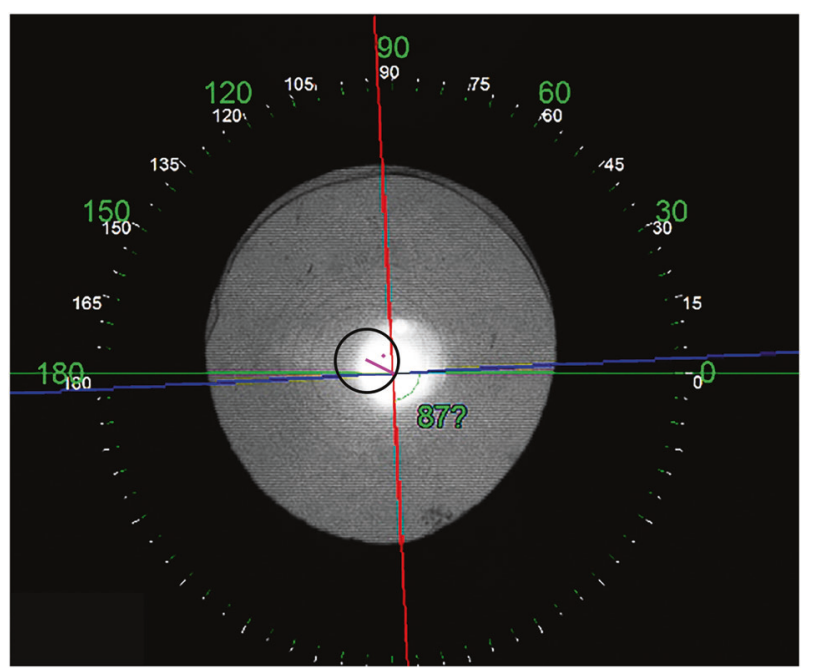

Fig. 1 The retroillumination analysis mode of the new generation of corneal topographer OPD-scan III. The rose-red line represents the decentration of the multifocal intraocular lens 
Table 1 The pre- and postoperative data for SN6AD1 and ZMB00 lenses

\begin{tabular}{llll}
\hline & SN6AD1 & ZMB00 & $P$ value \\
\hline Pre-operation & & & $95 \%$ CI \\
Age(year) & $68.35 \pm 8.55$ & $65.34 \pm 8.61$ & 0.121 \\
& $(71,47-79)$ & $(66,49-76)$ & 0.700 \\
Gender (male/female) & $17 / 25$ & $17 / 21$ & 0.345 \\
Eye (right/left) & $21 / 21$ & $15 / 23$ & 0.799 \\
Axial length (mm) & $23.82 \pm 1.09$ & $23.74 \pm 1.35$ & 0.515 \\
& $(23.62,22.21-25.72)$ & $(23.51,22.19-25.96)$ & -0.84 \\
UDVA (logMAR) & $0.62 \pm 0.31$ & $0.58 \pm 0.32$ & $-0.09,0.18$ \\
& $(0.52,0.22-1.40)$ & $(0.52,0.10-2.00)$ & 0.74 \\
Post operation & & & 0.326 \\
UDVA (logMAR) & $0.07 \pm 0.09$ & $0.12 \pm 0.10$ & $-0.04,0.10$ \\
CDVA (logMAR) & $(0.10,0.00-0.30)$ & $(0.10,-0.08$ to 0.30$)$ & 0.498 \\
DCNVA (logMAR) & $0.03 \pm 0.09$ & $0.07 \pm 0.08$ & $-0.05,0.08$ \\
& $(0.02,0.00-0.27)$ & $(0.02,-0.18$ to 0.24$)$ & 0.003 \\
\hline
\end{tabular}

Measurement data are presented as means \pm standard deviations, median, and range

$C I$ confidence interval, UDVA uncorrected distance visual acuity, $C D V A$ corrected distance visual acuity, DCNVA distance-corrected near visual acuity

Table 2 Tilt and decentration of SN6AD1 and ZMB00 lenses

\begin{tabular}{lllll}
\hline & SN6AD1 & ZMB00 & $P$ value & 95\% CI \\
\hline Intraocular tilt & $0.38 \pm 0.29$ & $0.74 \pm 1.07$ & 0.043 & $-0.70,-0.01$ \\
& $(0.32,0.04-1.56)$ & $(0.36,0.04-4.53)$ & 0.937 & $-0.08,0.07$ \\
Decentration: average (mm) & $0.35 \pm 0.17$ & $0.36 \pm 0.14$ & \\
Vertical (mm) & $(0.30,0.08-0.85)$ & $(0.33,0.15-0.77)$ & 0.556 & $-0.10,0.04$ \\
Horizontal (mm) & $0.26 \pm 0.15$ & $0.24 \pm 0.09$ & 0.376 \\
& $(0.23,0.00-0.60)$ & $(0.25,0.06-0.44)$ & $-0.04,0.07$ \\
\hline
\end{tabular}

Measurement data are presented as means \pm standard deviations, median and range.

$C I$ confidence interval

\section{Statistical analysis}

All measurement data are presented as means \pm standard deviations. Significance was tested with Student's $t$ test, the Mann-Whitney $U$ test, a paired $t$ test, and the $\chi^{2}$ test, with a significance level of $5 \%(P<0.05)$. The measurement data were analyzed with Pearson's correlation. All statistical analyses were performed with the SPSS 19.0 for Windows statistical analysis software (IBM-SPSS, Inc., Chicago, IL, USA).

\section{Results}

\section{Baseline data, visual acuity, and refraction}

The preoperative baseline information for these patients is shown in Table 1 . There were no statistically significant differences in age, sex, operated eye, axial length, or UDVA between the two groups (Student's $t$ test or $\chi^{2}$ test; all $P>$ $0.05)$. The UDVA of the patients treated with SN6AD1 or ZMB00 was significantly improved after surgery (Student's $t$ test; SN6AD1, $P<0.001$; ZMB00, $P<0.001)$. The SN6AD1 group achieved better postoperative UNVA than the ZMB00 group (Table 1). None of the patients in either group required any type of short- or long-distance correction in their daily lives after cataract surgery. 

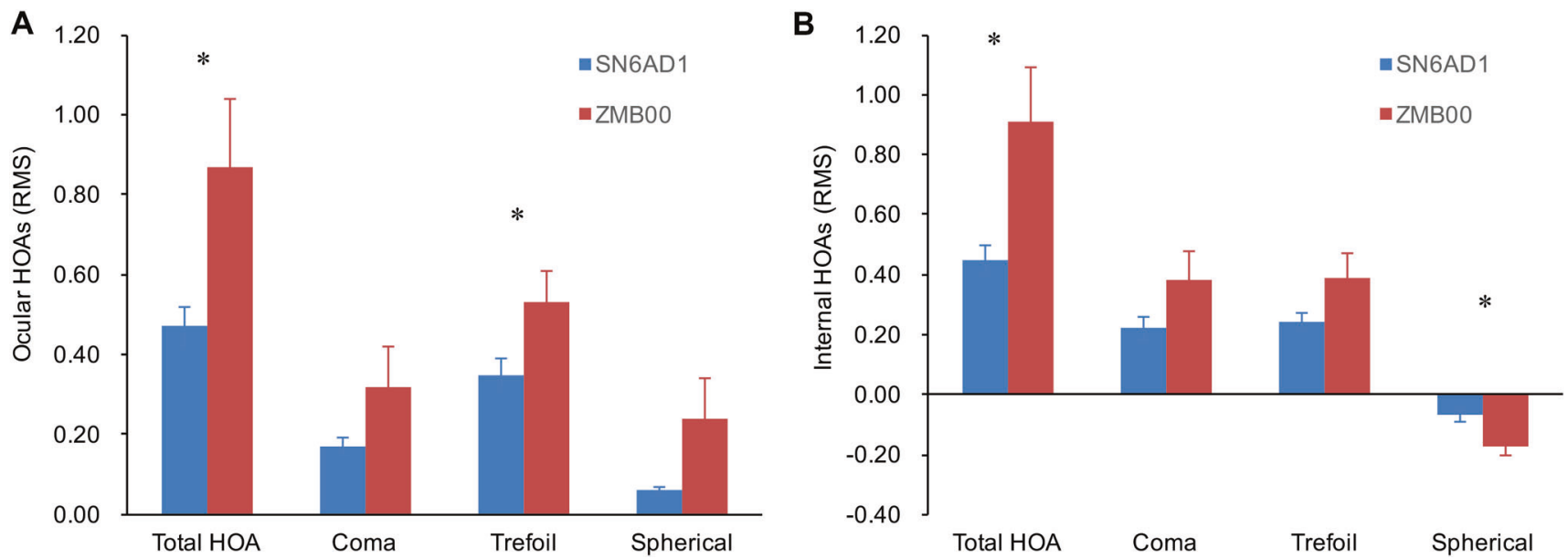

Fig. 2 Comparison of ocular (a) and internal (b) HOAs between SN6AD1 and ZMB00 group. (*Significant difference was found between the two groups, Student's $t$ test, $P<0.05)$. HOAs high-order aberrations, RMS root mean square

\section{Tilt and decentration of the MfIOLs}

The long-term average intraocular tilt was significantly lower in the SN6AD1 group than in the ZMB00 group, whereas the average decentration did not differ significantly in the two groups (Table 2). There was no significant difference in the vertical or horizontal decentration (Table 2).

\section{HOAs and correlation with tilt and decentration}

\section{HOAs}

The mean ocular and internal HOAs in the two groups, measured with OPD-Scan III, are shown in Fig. 2. The mean total ocular HOA, coma, and spherical aberration were significantly lower in the SN6AD1 group than in the ZMB00 group (Student's $t$ test, all $P<0.05$ ). The mean total internal HOAs, coma, trefoil, and spherical aberration were also significantly lower in the SN6AD1 group than in the ZMB00 group (Student's $t$ test, all $P<0.05$ ).

\section{Correlation with tilt and decentration}

In the SN6AD1 group, we found that intraocular tilt had a certain correlation with the total ocular HOAs (Pearson's correlation, $r=0.413, P=0.007)$, coma $(r=0.504, P=$ $0.001)$, and spherical aberration $(r=0.534, P<0.001)$, and to the total internal HOAs $(r=0.700, P<0.001)$, coma $(r$ $=0.702, P<0.001)$, trefoil $(r=0.502, P=0.001)$, and spherical aberration $(r=0.686, P<0.001)$, but there was no significant correlation between decentration and any type of HOA.

However, in the ZMB00 group, IOL decentration correlated positively with the total ocular HOAs (Pearson's correlation, $r=0.383, P=0.018)$, coma $(r=0.393, P=$
$0.015)$, and spherical aberration $(r=0.459, P=0.004)$, and with total internal HOAs $(r=0.455, P=0.004)$, coma $(r=$ $0.452, P=0.004)$, and spherical aberration $(r=0.484, P=$ 0.002 ). There was also a significant positive correlation between intraocular tilt and the total ocular HOAs (Pearson's correlation, $r=0.904)$, coma $(r=0.926)$, trefoil ( $r=$ $0.765)$, and spherical aberrations $(r=0.775$; all $P<0.01)$, and with the total internal HOAs $(r=0.936)$, coma $(r=$ $0.959)$, trefoil $(r=0.502)$, and spherical aberrations $(r=$ 0.686; all $P<0.01)$.

\section{Discussion}

Increasing numbers of middle-aged and older patients are involved in reading, computer use, and sports. Therefore, addressing pseudophakic presbyopia has become an important topic in the practice of cataract surgery and refractive lens exchange. MfIOLs are designed to improve the near vision of patients after cataract surgery and potentially allow the patient to read and write. Previous studies have shown that the implantation of SN6AD1 and ZMB00 MfIOLs can improve both distance and near vision $[4,10,11]$.

At present, there are three main types of MfIOLs, refractive, diffractive, and hybrid diffractive-refractive type lenses. The AcrySof ${ }^{\circledR}$ IQ ReSTOR ${ }^{\circledR}$ MfIOL SN6AD1 and the AMO Tecnis $^{\circledR}$ ZMB00 lenses are both single-piece foldable hydrophobic acrylic lens. The major difference between these two IOLs is their design and the optical surface. SN6AD1 has a refractive-diffractive hybrid design and an apodized diffractive anterior surface. The front surface is designed with a diffraction optic zone in the central $3.6 \mathrm{~mm}$ and the diameter of the central ring is 0.856 $\mathrm{mm}$. SN6AD1 provides bifocality with the addition of 3.0 
$\mathrm{D}$, and offers better long-distance visual acuity for larger pupils and better near-distance visual acuity for smaller pupils $[12,13]$. ZMB00 is a diffractive MfIOL with a diffractive posterior surface. It has 32 concentric rings, with a central ring of $1 \mathrm{~mm}$, and provides an additional $4.0 \mathrm{D}$, regardless of the pupil size $[2,11]$. Previous research has shown that $+3.0 \mathrm{D}$ is superior to $+4.0 \mathrm{D}$ in terms of the intermediate vision of the naked eye, the preferred reading distance, and the distance-corrected near vision under photopic conditions, and is more suitable for most people at a working distance $[4,5,13]$. These findings are similar to our results. However, under mesopic conditions, the design of the diffractive ring of the ZMB00 lens, which is distributed over the entire posterior surface, may provide better distance-corrected near vision because its light distribution is not affected by the pupil size [5].

However, the effect of MfIOLs is closely related to the centrality of the IOL after surgery [14]. Previous studies have shown that the decentration and tilt of MfIOLs can affect visual quality [14], such as the modulation transfer function (MTF) and the point spread function, but no clinical study has compared the differences in decentration and tilt between these two IOLs. Refractive MfIOLs may be affected by the dynamics of pupil size and decentration. However, diffractive MfIOLs are less dependent on pupil size and are more tolerant of the kappa angle and decentration. In the past, the measurement of decentration and tilt of IOLs has been mainly dependent on the instrument used, such as the EAS-1000 system, a Scheimpflug camera, ultrasound biomicroscopy, and anterior segment optical coherence tomography through to anterior segment tomography [15-18]. The IOL decentration value measured with these instruments is the distance between the center of the IOL and the center of the visua axis, whereas the OPD-Scan III shows the center of the visual axis and the diffraction ring using retroillumination, so the decentration value determined with OPD-Scan III could more accurately explain its influence on visual quality. Moreover, the center of the IOL can also be determined without pupil dilation because of the presence of a diffraction ring. Therefore, this apparatus has several advantages in measuring the decentration of diffractive MfIOLs. The intraocular tilt data acquired with the OPD-Scan III aberration measurements also suggest the tilt of the IOL to a certain extent. Therefore, one OPD-Scan III scan can provide the decentration and tilt data of an MfIOL, which simplifies the previously required complex calculation.

In terms of the decentration of MfIOL, our study suggests that the stability of SN6AD1 is better than that of the ZMB00 1 year after surgery, but no significant difference was found in the decentration values. This finding may arise from the same sizes of the SN6AD1 and ZMB00 MfIOLs. Several clinical studies have determined the decentration of
IOLs after cataract surgery, and the mean decentration in these studies was $0.30 \pm 0.16 \mathrm{~mm}$ [19-24]. The visual function of most MfIOLs may be affected by the amount of IOL decentration, the design of the MfIOL, and the pupil size. In a study of both diffractive and refractive MfIOLs, MTF and near images were affected by IOL decentration [25]. Another study evaluated the effects of different IOL designs on decentration, and demonstrated that more optically sophisticated IOL optics are more sensitive to decentration [26]. The visual performance of aberrationcorrecting IOLs is more affected by decentration than is the visual performance of aberration-free IOLs, whereas spherical IOLs are not affected by decentration at all.

Using OPD-Scan III aberration measurements, we found that the average intraocular tilt was significantly lower in the SN6AD1 group than in the ZMB00 group, which may related to the different design of the posterior optical surfaces of these two MfIOLs [5]. The STABLEFORCE ${ }^{\circledR}$ haptics of SN6AD1 may also deliver highly consistent compression forces, allowing axial stability in the lens capsule and anterior chamber stability.

Aberrometric analysis is a very important measurement when evaluating the outcomes of refractive surgeries. Several assessment instruments of high-order wavefront aberration may allow us to identify the optical quality and visual performance of the patient after cataract surgery [27]. In our study, we used OPD-Scan III for aberration measurements. The total and total internal HOAs were also lower in the SN6AD1 group than in the ZMB00 group 1 year after surgery, which may be related to the lower IOL tilt in the SN6AD1 group. IOL tilt determines the increase in HOAs, so poorer optical quality and limited performance are also related to worse refractive predictability. Previous in vitro tests indicated that the tilt of an MfIOL has a significant effect on visual quality [14], and our follow-up correlation analysis confirmed that MfIOL tilt correlated positively with the aberration value. However, in previous studies, there was no significant difference in HOAs between these two groups after a short follow-up period [4, 28]. A possible explanation involves the contraction of the capsular bag, which occurs more frequently with lenses made of soft materials. The ideal design of haptics and the stability of IOLs may increase the capacity to resist capsular bag contraction. We also found that the effect of decentration on postoperative aberrations was less than the effect of tilt after the implantation of these two MfIOLs, especially in the SN6AD1 group. This is similar to the results of previous studies, in which the effect of decentration was less pronounced on the diffractive MfIOL than on the refractive MfIOL.

In summary, the implantation of SN6AD1 and ZMB00 can significantly improve the visual acuity of patients, but the tilt was smaller in the SN6AD1 group than in the 
ZMB00 group 1 year after surgery, which may cause fewer HOAs and better visual quality.

\section{Summary}

\section{What was known before}

- The decentration and tilt of MfIOLs may significantly damage the designed optical quality, and consequently affect the patient's quality of life.

- There has been no comparison of long-term central stability after the implantation of AcrySof $^{\circledR}$ IQ ReSTOR $^{\circledR}$ SN6AD1 and AMO Tecnis $^{\circledR}$ ZMB00 MfIOLs.

- The generally accepted methods for evaluating the decentration and tilt of IOLs are the Purkinje imaging method and Scheimpflug image processing, both of which require other image processing software and complex calculations.

\section{What this study adds}

- The average decentration of SN6AD1 and ZMB00 did not differ significantly.

- The tilt of SN6AD1 was relatively smaller at 1 year after surgery, which may cause fewer HOAs and better visual quality.

- The new-generation corneal topographer OPD-Scan III provides tilt data based on the calculation of aberrations in one measurement, together with the eccentricity of IOLs determined with retroillumination.

\begin{abstract}
Acknowledgements This research was funded by research grants from the National Natural Science Foundation of China (grant no. 81470613, 81100653, 81670835, and 81270989), Shanghai High Myopia Study Group, International Science and Technology Cooperation Foundation of Shanghai (grant no. 14430721100), Shanghai Talent Development Fund (grant no. 201604), Shanghai Youth Doctor Support Program (grant no. 2014118), Outstanding Youth Medical Talents Program of Shanghai Health and Family Planning Commission, and scientific research program of Shanghai municipal health and Family Planning Commission (20174Y0186).
\end{abstract}

\section{Compliance with ethical standards}

Conflict of interest The authors declare that they have no conflict of interest.

\section{References}

1. Hayashi K, Ogawa S, Manabe S, Hirata A. Visual outcomes in eyes with a distance-dominant diffractive multifocal intraocular lens with low near addition power. $\mathrm{Br} \mathrm{J}$ Ophthalmol. 2015;99:1466-70.
2. Lubinski W, Gronkowska-Serafin J, Podboraczynska-Jodko K. Clinical outcomes after cataract surgery with implantation of the Tecnis ZMB00 multifocal intraocular lens. Med Sci Monit. 2014;20:1220-6.

3. Pedrotti E, Mastropasqua R, Passilongo M, Parisi G, Marchesoni I, Marchini G. Comparison of two multifocal intraocular lens designs that differ only in near add. J Refract Surg. 2014;30:754-60.

4. Chaves MA, Hida WT, Tzeliks PF, Gonçalves MR, Nogueira Fde B, Nakano CT, et al. Comparative study on optical performance and visual outcomes between two diffractive multifocal lenses: AMO Tecnis (R) ZMB00 and AcrySof (R) IQ ReSTOR (R) Multifocal IOL SN6AD1. Arq Bras Oftalmol. 2016;79:171-6.

5. Cillino G, Casuccio A, Pasti M, Bono V, Mencucci R, Cillino S. Working-age cataract patients: visual results, reading performance, and quality of life with three diffractive multifocal intraocular lenses. Ophthalmology. 2014;121:34-44.

6. Guyton DL, Uozato H, Wisnicki HJ. Rapid determination of intraocular lens tilt and decentration through the undilated pupil. Ophthalmology. 1990;97:1259-64.

7. Rosales P, De Castro A, Jimenez-Alfaro I, Marcos S. Intraocular lens alignment from purkinje and Scheimpflug imaging. Clin Exp Optom. 2010;93:400-8.

8. Jafarzadehpur E, Hashemi $\mathrm{H}$, Abdolahinia T, Yekta AA, Khabazkhoob M. Comparison of ocular aberrations in two hydrophobic and hydrophilic intraocular lenses. Eye Contact Lens. 2015;41:287-90.

9. McGinnigle S, Naroo SA, Eperjesi F. Evaluation of the autorefraction function of the Nidek OPD-Scan III. Clin Exp Optom. 2014;97:160-3.

10. Maurino V, Allan BD, Rubin GS, Bunce C, Xing W, Findl O. Quality of vision after bilateral multifocal intraocular lens implantation: a randomized trial--AT LISA 809M versus AcrySof ReSTOR SN6AD1. Ophthalmology. 2015;122:700-10.

11. Schmickler S, Bautista CP, Goes F, Shah S, Wolffsohn JS. Clinical evaluation of a multifocal aspheric diffractive intraocular lens. Br J Ophthalmol. 2013;97:1560-4.

12. de Vries NE, Nuijts RM. Multifocal intraocular lenses in cataract surgery: literature review of benefits and side effects. J Cataract Refract Surg. 2013;39:268-78.

13. de Vries NE, Webers CA, Montes-Mico R, Ferrer-Blasco T, Nuijts RM. Visual outcomes after cataract surgery with implantation of a $+3.00 \mathrm{D}$ or $+4.00 \mathrm{D}$ aspheric diffractive multifocal intraocular lens: comparative study. J Cataract Refract Surg. 2010;36:1316-22.

14. Montes-Mico R, Lopez-Gil N, Perez-Vives C, Bonaque S, FerrerBlasco T. In vitro optical performance of nonrotational symmetric and refractive-diffractive aspheric multifocal intraocular lenses: impact of tilt and decentration. J Cataract Refract Surg. 2012;38:1657-63.

15. Choi SK, Kim JH, Lee D, Park SH, Maeda N, Ma KJ. IOL tilt and decentration. Ophthalmology. 2010;117:1862. 1862.e1861-1864

16. Kranitz K, Mihaltz K, Sandor GL, Takacs A, Knorz MC, Nagy ZZ. Intraocular lens tilt and decentration measured by Scheimpflug camera following manual or femtosecond lasercreated continuous circular capsulotomy. J Refract Surg. 2012;28:259-63.

17. Wang $\mathrm{X}$, Dong J, Wang $\mathrm{X}$, Wu Q. IOL tilt and decentration estimation from 3 dimensional reconstruction of OCT image. PLoS ONE. 2013;8:e59109.

18. Zhao YE, Gong XH, Zhu XN, Li HM, Tu MJ, Coursey TG, et al. Long-term outcomes of ciliary sulcus versus capsular bag fixation of intraocular lenses in children: an ultrasound biomicroscopy study. PLoS ONE. 2017;12:e0172979.

19. Baumeister M, Neidhardt B, Strobel J, Kohnen T. Tilt and decentration of three-piece foldable high-refractive silicone 
and hydrophobic acrylic intraocular lenses with 6-mm optics in an intra-individual comparison. Am $\mathrm{J}$ Ophthalmol. 2005;140:1051-8.

20. de CA, Rosales P, Marcos S. Tilt and decentration of intraocular lenses in vivo from Purkinje and Scheimpflug imaging. Validation study. J Cataract Refract Surg. 2007;33:418-29.

21. Jung CK, Chung SK, Baek NH. Decentration and tilt: silicone multifocal versus acrylic soft intraocular lenses. J Cataract Refract Surg. 2000;26:582-5.

22. Oshika T, Sugita G, Miyata K, Tokunaga T, Samejima T, Okamoto $\mathrm{C}$, et al. Influence of tilt and decentration of scleral-sutured intraocular lens on ocular higher-order wavefront aberration. $\mathrm{Br} \mathbf{J}$ Ophthalmol. 2007;91:185-8.

23. Taketani F, Matuura T, Yukawa E, Hara Y. Influence of intraocular lens tilt and decentration on wavefront aberrations. J Cataract Refract Surg. 2004;30:2158-62.
24. Taketani F, Yukawa E, Yoshii T, Sugie Y, Hara Y. Influence of intraocular lens optical design on high-order aberrations. J Cataract Refract Surg. 2005;31:969-72.

25. Soda M, Yaguchi S. Effect of decentration on the optical performance in multifocal intraocular lenses. Ophthalmologica. 2012;227:197-204.

26. Eppig T, Scholz K, Loffler A, Messner A, Langenbucher A. Effect of decentration and tilt on the image quality of aspheric intraocular lens designs in a model eye. J Cataract Refract Surg. 2009;35:1091-1100.

27. Alio JL, Peña-García P, Pachkoria K, Alio JL 2nd, El Aswad A. Intraocular optical quality of phakic intraocular lenses: comparison of angle-supported, iris-fixated, and posterior chamber lenses. Am J Ophthalmol. 2013;156:789-99.

28. Yang HC, Chung SK, Baek NH. Decentration, tilt, and near vision of the array multifocal intraocular lens. J Cataract Refract Surg. 2000;26:586-9. 